Europe PMC Funders Group

Author Manuscript

Sleep Med Rev. Author manuscript; available in PMC 2010 April 15.

Published in final edited form as:

Sleep Med Rev. 2010 April ; 14(2): 97-105. doi:10.1016/j.smrv.2009.04.003.

\title{
Sleep in disorders of consciousness
}

\author{
Victor Cologan $^{\mathrm{a}, \mathrm{e}}$, Manvel Schabus ${ }^{\mathrm{a}, \mathrm{b}, \mathrm{f}}$, Didier Ledoux ${ }^{\mathrm{c}, \mathrm{g}}$, Gustave Moonen $^{\mathrm{d}, \mathrm{h}}$, Pierre \\ Maquet $^{a, d, i}$, and Steven Laureys ${ }^{a, d,{ }^{*}}$ \\ a Coma Science Group, Cyclotron Research Center, University of Liège, Belgium \\ b Division of Physiological Psychology, University of Salzburg, Austria \\ ${ }^{c}$ Department of Intensive Care Medicine, Sart Tilman University Hospital, Liège, Belgium \\ d Department of Neurology, Sart Tilman University Hospital, Liège, Belgium
}

\section{SUMMARY}

From a behavioral as well as neurobiological point of view, sleep and consciousness are intimately connected. A better understanding of sleep cycles and sleep architecture of patients suffering from disorders of consciousness (DOC) might therefore improve the clinical care for these patients as well as our understanding of the neural correlations of consciousness. Defining sleep in severely brain-injured patients is however problematic as both their electrophysiological and sleep patterns differ in many ways from healthy individuals. This paper discusses the concepts involved in the study of sleep of patients suffering from DOC and critically assesses the applicability of standard sleep criteria in these patients. The available literature on comatose and vegetative states as well as that on locked-in and related states following traumatic or non-traumatic severe brain injury will be reviewed. A wide spectrum of sleep disturbances ranging from almost normal patterns to severe loss and architecture disorganization are reported in cases of DOC and some patterns correlate with diagnosis and prognosis. At the present time the interactions of sleep and consciousness in brain-injured patients are a little studied subject but, the authors suggest, a potentially very interesting field of research.

\section{Keywords}

Consciousness; Coma; Vegetative state; Minimally conscious state; Locked-in syndrome; Brain injury; Sleep; Arousal; Polysomnography

\section{Introduction}

An accurate and reliable assessment of arousal and awareness in disorders of consciousness (DOC) is of major importance for patients' clinical management. Arousal can be regarded as the level of consciousness while awareness, its content (Fig. 1). Progress in intensive care has led to an increase in the number of patients who survive severe acute brain damage. Although some of these patients recover from their coma within the first days or weeks after

\footnotetext{
(c) 2009 Elsevier Ltd. All rights reserved.

*Correspondence to: Coma Science Group, Cyclotron Research Center, Sart Tilman B-30, 4000 Liege, Belgium. Tel.: +32 436623 04; fax: +32 436629 46. steven.laureys@ulg.ac.be (S. Laureys)..

eTel.: +32436623 62; fax: +32 436629 46. vcologan@student.ulg.ac.be (V. Cologan)

f Tel.: +43 6628044 5143; fax: +43 6628044 5126. manuel.schabus@ @bg.ac.at (M. Schabus)

gTel.: +32 436674 95; fax: +32 436688 98. dledoux@ @ chu.ulg.ac.be (D. Ledoux)

hTel.: +32 436672 55; fax: +32 436674 99. g.moonen@ @hu.ulg.ac.be (G. Moonen)

'Tel.: +32 436623 67; fax: +32 436629 46. pmaquet@ulg.ac.be (P. Maquet)
} 
the injury, some permanently lose all brain function (i.e., brain-death) or motor function (i.e., locked-in syndrome, LiS), whereas others will progress towards a state of wakeful unawareness (i.e., vegetative state, VS). Patients who recover from the latter condition typically progress through different stages before reaching partly recovering (i.e., minimally conscious state, MCS) or before fully recovering consciousness.1

Clinical practice has shown how challenging it is to identify unambiguous signs of conscious perception both of the environment and of self in DOC sufferers. In consequence misdiagnoses of VS and LiS are common ( $\sim 35 \%$ and $\sim 50 \%$ respectively; for review $\left.\operatorname{see}^{2}\right)$. Bedside assessment of residual brain function in patients who are severely brain damaged is difficult because motor responses may be very limited or inconsistent. DOC have also been described according to electrophysiological criteria and, more recently, those of functional neuroanatomy.1 Neurophysiological studies rely mainly on the evaluation of continuous bedside EEG3 or event-related potentials 4 and can contribute to establishing diagnosis and prognosis. Functional neuroimaging brings a pathophysiological point of view and is an additional diagnostic factor. It can, for example, provide an objective measure of the regional distribution of cerebral activity at rest and under various conditions of stimulation.1 However the use of such techniques in DOC is methodologically complex, needs careful analysis and interpretation, and does not permit establishing prognoses at the present time. Identifying markers of recovery in DOC is even more challenging. Prognosis mostly depends on age, etiology and the time lapse since brain injury along with the Glasgow Coma Scale subscores (GCS, the gold standard behavioral scale of responsivity in DOC), serum levels of neuron-specific enolase (for anoxic coma), somesthesic evoked potentials and EEG of the patient.

Sleep is a behavior usually characterized by the adoption of a typical posture and the absence of response to external stimuli due to transient but reversible periods of unconsciousness which, in healthy individuals, are accompanied by well-defined EEG changes.5 In DOC however, the operational electrophysiological definition of wakefulness and sleep is problematic because oscillations recorded by EEG no longer reflect the same cellular mechanisms as in normal physiological sleep. For example large amplitude slow waves do not necessarily indicating deep non-rapid eye movement (NREM) or "slow-wave" sleep (SWS) as they do in normal sleeping individuals. Sleep stage criteria are undefined in DOC and therefore to establish sleep staging, criteria need to be explicitly defined.

The present paper focuses on the importance of neurophysiology for the evaluation of the biological rhythms in DOC and their relationships with diagnosis and prognosis. In the first section we will discuss the importance of evaluating biological rhythms such as the circadian rhythm to support the existence of sleep-wake cycles in DOC. We will then address the difficulty in applying standard sleep scoring systems 5 to these patients. Next we will review the available data on sleep according to the different states of consciousness, that is to say brain-death, coma, VS, the recovery of consciousness and LiS. No study on MCS, which was only introduced recently, 45 could be found in the available literature.

\section{Studying sleep in DOC: conceptual \& methodological issues}

\section{Conceptual issues}

Although it is well-known that sleep abnormalities are extremely common in critically ill patients, 6,7 their mechanisms remain poorly understood and their fine-grained characterizations are still unknown. Firstly we must highlight the differences found in DOC sufferers compared to that of healthy individuals. From a behavioral point of view, normal sleep is usually preceded by the search for a safe place and a progressive but reversible 
decrease in response to external stimuli and a decrease in motor activity. In DOC, assessing these behavioral criteria is challenging and rarely conclusive.

From a physiological point of view, normal sleep is associated with well described cycles, stages, arousals and micro-structures (e.g., K-complexes, spindles). The general limitations of the Rechtschaffen \& Kales criteria5 in clinical contexts have been summarized elsewhere. 8 In DOC the existence of such polysomnographic elements is a matter of debate. As we will discuss, some authors suggest their persistence in some more "evolved state", whereas others refute this.

Another characteristic aspect of sleep is its regulation by homeostasic and circadian factors. In DOC the available evidence for such regulators is scarce. In 10 patients in traumatic persistent VS it was shown that heart-rate and blood pressure did not show the normally observed nocturnal decrease during night hours.9 Perhaps this was because patients also slept during the day and might not have been sleeping at the recording times. Another study with 16 persistent VS patients (12 postvascular encephalopathy) showed significant circadian changes in body temperature and urinary excretion of hormones and sodium but observed no changes in blood pressure or pulse rate. The authors also reported significant circadian variations in blood pressure and pulse rate in five out of six patients who showed "a simple organized response to noxious external stimuli". These findings suggests that the preservation of circadian rhythm may vary with the severity of brain damage or with patients' "state of consciousness". 10 One study held at an intensive care unit11 revealed the loss of circadian secretion of melatonin in sedated "unresponsive" patients with severe sepsis.

It appears that the very existence of sleep and its characterization in DOC is a most challenging issue as these patients do not show the normal behavioral, physiological and regulatory signs of sleep. Circadian and homeostasic factors are potent modulators of sleep and it is therefore surprising that previous studies have not aimed to thoroughly investigate their existence and possible diagnostic and prognostic value before focusing on any macroor micro-structural sleep changes. To this end the assessing of modifications in hormonal plasma levels (e.g., melatonin, cortisol), temperature, along with those of blood pressure, heart-rate and actimetry monitoring, should be performed first to accurately outline circadian variations in DOC. Support for the presence of homeostasic sleep regulation in DOC could be provided by wake extension protocols and the comparing of brain EEG before and after a period during which patients would be prevented from closing their eyes using external stimulation.

Earlier studies on coma suggested that the presence of EEG patterns resembling sleep may be reliable markers for a favorable outcome.12,13 It was reported that sleep patterns continue to become more complex during rehabilitation therapy, paralleling patients' cognitive recovery.14 Some authors have used standard Rechtschaffen \& Kales sleep criteria to analyze the polysomno-graphic (PSG) data in DOC.13,15 However, as many forms of brain damage may result in a relatively similar clinical state of unconsciousness and as modification of brain activity in DOC may substantially differ from physiological sleep patterns described by Rechtschaffen \& Kales, those criteria are probably not applicable for sleep staging in severely brain damaged patients. We suggest that these scoring criteria need to be adapted for the study of sleep-wake patterns in pathological DOC. To this end the visual adaptive scoring system (which describes vigilance levels8), or the analysis of microarousals16 may be useful alternatives.

In conclusion, the study of sleep is of particular interest in DOC with various different etiologies as it can provide relationships between some graphoelements and functional 
neuroanatomy. Whereas waking patterns, in non-comatose patients, only indicate the persistence of the reticular activating system. As an example, spindles may reflect the preserved functional integrity of the thalamus; SWS and REM sleep may reflect residual functioning of brainstem nuclei; and the circadian organization of sleep patterns are informative of residual hypothalamic functioning. Nevertheless the analysis of waking EEG, cleaned for muscular artifacts (which are often exacerbated in DOC) and eye-blinks may also yield useful diagnostic and prognostic information.

\section{Methodological issues}

In intensive care units the environmental factors are often not conducive to sleep. For example, mechanical ventilation can cause considerable sleep disruption. Research on sleep in DOC sufferers needs to control factors such as ventilator modes. Further factors contributing to sleep abnormalities in intensive care units include acute systemic illness, pain and discomfort or increased alertness induced by continuous exposure to light, noise and nursing activities. Importantly, sleep deprivation is known to have a negative impact on behavior but also on immune and endocrine functions.17 It may also induce sympathetic activation and elevation of blood pressure, which in turn can be a factor in patient morbidity. 7 On the other hand circadian conditions in rehabilitation centers or at home are generally more favorable and sleep disturbance may be minimized. It is therefore suggested that optimizing sleep efficiency in order to promote optimal neurobehavioral functioning is an important issue that clinicians may want to address.

PSG is the only tool allowing reliable evaluation of sleep in DOC but recording good quality signals is very challenging due to artifacts caused by perspiration, thermal dysregulation, skin and skull lesions, electrical artifacts from life-supporting devices and other signs related to vegetative or autonomous function "storms" in this patient population. When looking at the basic EEG structure, the first examination made is to test for reactivity (i.e., a change in EEG caused by external sensory stimulation). Painful or auditory stimuli are usually used for this purpose and the presence of EEG reactivity often indicates a less severe level of coma.3

Another evaluation, although rarely used in the field of coma, concerns arousal organization which is linked to sleep's micro-organization. Arousal can be defined as simultaneous changes in the EEG along with autonomic system and somatic motor activity.16 Compared to healthy individuals, patients with DOC do not exhibit the normal arousal alternations also known as "standard cyclical alternating patterns". 18 In these patients, changes in brain activation may be very slow, lasting a number of seconds or even minutes, and are not always rhythmic. These arousal alternations are often more extreme than normal and may even be life-threatening, especially those occurring in the vegetative system (e.g., involving cerebrospinal fluid pressure increases19). The contrast between the extreme changes in sleep microstructure seen in the damaged brain and the diffident ones in the normal brain suggests a process in which arousal control mechanisms have been altered in DOC.

The well-known advantages of EEG recording include its high temporal resolution and its non-invasive nature, along with its portability and low cost. EEG is also useful in excluding non-convulsive status epilepticus and in providing a rough but objective guide as to the degree of cortical and subcortical dysfunction in DOC.20 Its main limitation is the lack of specificity (e.g., diffuse slowing of background rhythms is seen in various encephalopathies regardless of etiology3). Another limitation of EEG is its low spatial resolution. With the possible exception of high-density EEG, source reconstruction is possible but it never equals the spatial resolution of functional magnetic resonance imaging (fMRI) and remains more difficult for use on deep brain structures. The EEG can also be contaminated by uncontrolled eye movements and muscle activation which regularly occurs in DOC. Sophisticated 
correction methods, using for example Independent Component Analysis, may be necessary in order to obtain clean EEG data exploitable for scientific and clinical interpretation.

\section{Brain-death}

Until recently, studying sleep in brain-dead patients seemed pointless as the EEG is invariantly isoelectrical in this condition. 1 However a case-report recently described sleeplike periodic leg movements (PLM) in brain-death.21 Following a massive intracranial hemorrhage, a 50 year-old man remained comatose for $24 \mathrm{~h}$ and then was examined for $12 \mathrm{~h}$ before being diagnosed brain dead. Over this period leg movements, very similar to natural PLM during sleep in terms of both behavioral characteristics and periodicity, were observed for $30 \mathrm{~h}$. Several earlier reports have described PLM during sleep although patients were suffering from severe brainstem 22 and spinal cord lesions.23 This case-report suggests that, although the anatomical substrate involved in the generation of PLM remains unclear, PLM in sleep and in brain-death may share a common mechanism. The authors speculate that the brainstem looses its control over the descending spinal pathway during classical PLM in sleep as it does in brain-death.

\section{Coma}

In coma the EEG often shows a generalized slowing in the delta or theta range. Other EEG patterns that can be encountered include alpha-coma, burst-suppression and epileptiform activity.3 No differentiation between normal sleep and pathological slow waves has been illustrated in the literature. However continuous delta activity in coma should not be mistaken for normal SWS. Coma can be considered as a dysregulation of the brain's arousal system caused by diffuse brain damage or by focal brainstem lesions. The arousal system can be considered at three levels: (1) an upper level encompassing cerebral cortex and white matter; (2) a middle level encompassing thalamus and upper brainstem; and (3) a lower level encompassing lower midbrain and pons. Although the upper level does not seem to be necessary to sustain arousal and is instead linked with awareness, brain damage at any or all these levels may result in coma or various arousal and EEG alterations.3,19

The rare literature concerning sleep in coma mainly deals with spindle coma (SC). SC is an EEG pattern in which physiologic sleep elements, resembling sleep spindles in the 9-14 Hz range, vertex sharp waves and K-complexes, are superimposed on a background of delta ( $<4$ $\mathrm{Hz}$ ) and theta $(4-8 \mathrm{~Hz})$ activity. The occurrence of such physiological patterns resembling NREM sleep were first studied by Chatrian et al.13 who described NREM elements such as slow waves and spindles in traumatic DOC. Further work showed that non-traumatic causes, mainly metabolic, infectious and hypoxic encephalopathy 24 or syncope 25 may also result in SC. However, in a study of 370 comatose patients of different etiology, less than $6 \%$ showed a SC pattern.26 In reviewing the literature, Kaplan and colleagues 27 postulated that SC could be a more benign form of coma (with $25 \%$ mortality rate) and that the combination with EEG reactivity to noxious stimuli heralds a favorable outcome, independent of the etiology.

Some earlier studies have also indicated that sleep spindles may carry prognostic information. It was subsequently shown that the presence of spindle activity after hypoxic or anoxic injury does not always indicate a good outcome. However the absence of spindles or EEG background reactivity does predict a poor outcome.28 A more recent study supports these findings in comatose children and concludes that the reappearance of sleep patterns and sleep spindles is sign of good prognosis. In traumatic coma, these sleep elements are more frequently observed than in anoxic cases. 29 
Spindle activity may depend on the time since coma onset. Such activity occurred in $91 \%$ of cases if recorded within 1-2 days after injury and in 30\% of more prolonged cases (3-12 days). 30 A clear distinction between a coma characterized by a continuous spindle pattern and a coma with occasional sleep cycles and spindles has not yet been reported. It is to be noted that the former condition represents SC stricto sensu and is rare $\left(5 \%{ }^{27}\right)$. The topography and temporal dynamics of spindle activity, as well as the presence of different spindle types in coma, have not yet been thoroughly investigated. It is assumed that spindle coma represents a combination of physiological sleep and coma, the latter accounting for the failure of arousal. In humans, the pathophysiological mechanism of SC is presumed to be the preservation of pontine raphe nuclei and thalamocortical circuits subserving sleep spindle activity, together with the impairment of ascending reticular activating pathways at the midbrain level that maintain wakefulness.31-33 Future research should agree on a common definition of PSG evaluation over the course of a coma.

Over and above the SC topic, a few authors used PSG in order to determine the severity of brain damage in coma. A study in 138 unselected patients recorded within seven days of head injury showed prognostic differences based on the assessment of arousal related phasic activity involving EEG, motor and autonomic changes (Table 1, left column).34 PSG data were superior predictors of outcome when compared to the Glasgow coma scale (GCS) scores at the time of recording. Similarly, the predictability of different levels of sleep-wake organization in traumatic coma (Table 1, right column) was compared to other indexes such as neuroradiological findings, age or GCS scores. 35 The presence of sleep-like patterns rather than GCS scores was shown to be indicative of a better outcome. NREM sleep elements such as K-complexes and sleep spindles as well as rapid eye movement (REM) sleep elements alternating with NREM sleep elements were also indicators of a better outcome. In contrast, a poor outcome was indicated for patients who had only monophasic EEG or a cyclic alternating pattern with absence of sleep elements.

In conclusion, the more the comatose patient's brain activity resembles normal healthy sleep, the better the prognosis. It appears that there may exist indicators of good outcome such as spindles, phasic arousal activity and the conservation of sleep stages. This would justify the use of PSG in the clinical routine. Disruptions of sleep patterns and NREM phasic events (e.g., spindles) are often found in the early stage of coma. Given that human spindle generators are located in the thalamus, it is tempting to hypothesize that the absence of spindles in coma results from the interruption of either the ascending reticular thalamocortical pathway or of thalamocortical loops. The absence of sleep-wake cycles seems associated with brainstem or hypothalamus dysfunction and preliminary evidence suggests this may be associated with a poor outcome.

The difficulty in interpreting the literature data on SC resides in the variable methodology of electrophysiological recordings and the difficulty of comparing clinical entities, etiologies and outcomes. Prolonged PSG has hardly been employed in DOC and the use of EEG has been variable in terms of the time since the injury occurred, time and duration of recording and the lack of standardized stimulation conditions. The study of sleep organization in coma remains uncommon despite its possible prognostic interest and prolonged PSG is not performed as part of the clinical routine assessments in intensive care units. Most published studies involve a small number of patients with varying and sometimes suboptimal methodology. Nor do they take into account possible confounding factors at the intensive care units. Given that comatose patients, by definition, cannot be clinically aroused (based on the absence of eye-opening), most studies do not consider possible cyclic changes in brain activation. 


\section{Vegetative state}

Only a few research groups have written about sleep in VS (Table 2). Compared to the healthy control group, only minor sleep alterations were found in 9 traumatic patients with good outcome and no sleep patterns were found in one permanent VS patient.36 In another study it was brought to light that patients "in the last remission stages" went through all sleep stages with an increase of total sleep time and the amount of all sleep stages in comparison to patients "in the first remission stages" of VS.37 As discussed above, spindle activity may be related to both injury severity and recovery. Evidence of spindles, although always reduced in density and duration, was found in 11 out of 20 traumatic and 3 out of 10 hypoxic VS patients.38 In addition, the authors showed an increase of spindle density from 5 to 12 per min paralleling the clinical recovery of traumatic patients. As for coma, these results suggest spindles as potential markers of good outcome in VS but more studies are warranted.

Other authors focused on REM sleep in VS patients. An earlier study has shown the occurrence of nystagmus in wakeful and REM stages of 6 vegetative patients. 39 The same authors also showed both a degradation of REM sleep and specific phasic events such as the number of rapid eye movements in 11 traumatic VS patients. 15 These findings might reflect possible damage or dysregulation in the pedunculopontine tegmental cholinergic structures in VS. Nevertheless, other phasic events such as sleep-related erections 40 seem to be preserved. At present, no correlation between REM parameters and recovery from VS could be shown.

Although brainstem mechanisms responsible for sleep-wake cycles and for the appearance of REM sleep seem largely preserved in VS, the marked reduction in phasic REM activities in patients suggests that other brainstem mechanisms might be affected. As neural generators of phasic and tonic REM sleep components are localized in the pontine tegmentum, 41 the decrease in the densities of the REMs and muscle twitches during REM sleep may reflect possible damage to the cholinergic mechanisms of the pedunculopontine tegmentum. In animal studies, these have been demonstrated to be critical for REMs and ponto-geniculo-occipital waves, but not for EEG desynchronization and muscular atonia.42 Nevertheless, lesions in the pedunculopontine tegmentum do not affect the duration of REM sleep episodes suggesting that non-cholinergic cells also play a critical role in the maintenance of REM sleep. Indeed, serotoninergic cells in the dorsal raphe and noradrenergic cells in the locus coeruleus appear to be implicated in the suppression of phasic events.41,42 Interestingly, an fMRI study of early post-traumatic VS patients showed that lesions in the dorsolateral brainstem and corpus callosum are predictive of nonrecovery. 43

Overall, in the reviewed literature, the provided details on studied patients remain insufficient and standardized methods were rarely used to assess behaviorally the level of consciousness. One study reported the absence of sleep-wake cycles in 4 out of $12 \mathrm{VS}$ patients.44 This may pose a problem with regard to our clinical criteria of VS, as the presence of behaviorally assessed sleep-wake cycle is currently needed for its diagnosis. In summary, large cohort studies of well-documented VS patients are of great necessity to (1) provide a better understanding of the presence (or absence) of sleep-wake cycles in vegetative (and minimally conscious) states, and (2) to reveal more detailed relationships between the various brain injuries, sleep parameters and their clinical outcome.

\section{Recovery from coma}

As the minimally conscious state criteria were only defined in 2002,45 no article concerning sleep in MCS patients could be identified. We have thus reviewed literature on patients who 
recovered consciousness after injury and who were studied in neurorehabilitation centers or in their homes.

George and colleagues were amongst the first to use longitudinal EEG recordings in severely brain damaged patients. They showed disturbances in wakefulness during sleep and REM sleep during recovery from coma.46,47 Symptoms of insomnia were reported by $70 \%$ and $37 \%$ of the patients $(n=60)$ at 1.5 and 3 months after coma recovery, respectively.48 Other authors reported insomnia complaints shortly after recovery (at 3.5 months) and predominant hypersomnia complaints 29 months after recovery from traumatic coma. 49 Surprisingly, a longitudinal study of 130 patients of all etiologies found that the more severe the brain injury the less likely patients exhibited such sleep disturbances.50 Otherwise, early studies in DOC indicated a high correlation between cognition and REM sleep improvement in 7 traumatic patients 9 months after regaining consciousness. 14

Other studies brought to evidence abnormal "multiple sleep latency test" values, sleep disruption, sleep-disordered breathing and daytime sleepiness in 184 somnolent patients who recovered from traumatic coma.51 In addition, "multiple sleep latency test" alterations were positively correlated with injury severity. In line with these findings, other authors reported hypersomnia in $47 \%$ of brain damaged patients $(n=71)$ and also noted a relatively high prevalence of sleep apnea-hypopnea syndrome and periodic limb movement disorder.52 It was also shown that insomnia is linked with both the presence of milder traumatic brain injuries as well as depression.53 These authors suggested that the determinants of insomnia may differ from the acute to post-acute phase, with neurological factors playing a primary role early in the recovery process and psychosocial factors occurring at later stages. They next showed that $42 \%$ of the post-comatose population were found to suffer from insomnia. 54 Surprisingly, poor sleep quality and insomnia were much greater for the rehabilitation comparison group (e.g., spinal cord injury and musculoskeletal diseases). This may suggest that the comparison group might have undergone more severe psychosocial stressors (like anxiety and depression) leading to increased sleep disturbances but the underlying reasons remain unclear.

A series of studies by Ouellet and Morin focused on insomnia after traumatic brain injury.55 Comparing subjective (i.e., sleep diary) and objective (i.e., nocturnal PSG) sleep measures, these authors showed that brain-injured patients have a tendency to overestimate their sleep disturbance, as normal insomniacs often do. They also described an abnormally high proportion of stage 1 sleep, long lasting awakenings, as well as a shorter REM sleep latency. 56 They next analyzed 452 recovered traumatic coma survivors and showed that $80 \%$ reported insomnia symptoms or syndrome, the majority of the latter being untreated, and that the associated risk factors were milder traumatic brain injury, depression and pain.57 They also published a single-case study about cognitive behavioral therapy for insomnia.58 The effects of this treatment revealed a normalization of total sleep time and wake after sleep onset, of sleep onset latency, as well as sleep efficiency. The majority of gains were well maintained at 1- and 3-month follow-up assessments, suggesting that sleep disturbances in recovered traumatic coma can be treated with standard nonpharmacological interventions.

In conclusion, patients who recover consciousness after coma may undergo various sleep disturbances (see Table 3) including insomnia, hypersomnia, sleep pattern and latency modifications - the latter often being associated with fatigue problems. One study suggests that REM density may be regarded as an index of cognitive recovery.14 Slow-wave sleep seems relatively preserved compared to the other vigilance states but thalamocortical dysfunction causing sleep disturbances may persist after recovery from coma. These disturbances are often reported as being independent from etiology but almost all reviewed studies included traumatic injuries. Additional data is needed to investigate sleep 
disturbances in non-traumatic patients, the problem being that they regain consciousness more rarely. The described sleep disturbances are found to be strongly correlated with injury severity, and less with age, sex, mood and interval between injury and investigation. Injury severity, however, is often a subjective descriptive variable and fails to take into account injury localization and extension, coma duration and other neurological confounding factors. Until now most of the authors do not specify the duration of coma and the interval between injury and investigation. In general, the reviewed literature employs different PSG setups, preventing valid comparisons between studies. Longitudinal studies using PSG of minimum $24 \mathrm{~h}$ duration as well as standardized behavioral scales in clearly documented patient populations would permit a better understanding of sleep disturbances following long-term recovery after acute brain injury.

Etiological factors of sleep disorders in the context of brain injury are manifold and include factors that predispose some patients to develop those disorders (presence of a psychiatric disorder, increased physiological vulnerability), factors that trigger disorders onset (psychological reactions to brain injury related stressors, side effects of treatments, pain), and factors that are instrumental in the maintenance of disorders over time (maladaptive sleep behaviors, dysfunctional attitudes and beliefs about sleep). Fatigue, mood disturbances, and exacerbated cognitive deficits are among the possible consequences of sleep alterations in recovered DOC patients, although much more research is needed to document the effects of these disturbances on patients' cognitive functioning. Despite the high prevalence of sleep problems and their likely negative consequences, there are very few studies concerning their treatment. Although the prescription of hypnotic medications may be an effective method to treat sleep disorders, long-term use is often problematic. Considering that sleep alterations are very prevalent in DOC even several years after the injury, it may be important to offer alternative or combined pharmacological and psychological treatment options.58 Available data collected in otherwise healthy individuals suggest that cognitive behavioral therapy would represent a promising therapeutic option and could successfully be integrated in DOC patients' routine care. The same holds for alternative treatments based on neurofeedback.59 Early evaluation and treatment of sleep disturbances in patients who have recovered from coma should be considered as an integral part of their rehabilitation process.

\section{Locked-in syndrome}

The literature on sleep in locked-in syndrome (LiS) patients is scarce and relatively old (see Table 4). Guilleminault and colleagues were amongst the first to work on sleep in LiS.60 They described a case with unspecified brainstem lesion having hyposomnia without REM sleep abnormalities. According to subsequent studies, it appears that the more extended the pontine lesion, the more pronounced the sleep disturbances, especially for REM sleep. The lesion severity increases in case of bilateral or dorsal extension, and of tegmentum involvement, especially if the raphe nucleus also is lesioned. The observed spectrum of altered sleep in LiS can vary from almost normal sleep patterns61,62 to severe sleep quantity decrease,63-65 disorganized NREM sleep61,62,66 and stage 463 or REM absence. $61,62,66,67$ In rare cases, LiS patients presented only minor sleep abnormalities even when having severe pontine lesions and persistent neurological deficits.68 Note that all except 2 cases of the reviewed literature are of vascular (ischemic or hemorrhagic) origin and that the "unresponsiveness" state preceding the LiS diagnosis is not always clearly documented.

Further studies providing detailed clinical and PSG data are necessary in order to better understand the relationship between brainstem lesions and sleep disturbances in LiS. Finally, as we mentioned for recovered patients, careful attention must be given to the larger 
psychosocial context in which sleep disorders may emerge, particularly to the role of emotional disturbances which are certainly very important in LiS patients.

\section{Conclusion}

In summary, DOC are often linked with alterations of sleep. In the comatose state, the patient by definition shows no eye-opening and no sleep-wake cycle. However, a variety of arousal responses have been shown and, in certain cases, NREM and REM graphoelements may emerge. In the VS, patients by definition show transient periods of eye-opening and sleep-wake cycles. However, very few studies provided empirical evidence for their residual sleep architecture. Most of the existing literature deals with spindling activity and REM changes related to recovery from coma. It should be emphasized that some of the patients being reported as VS may in fact be MCS (as the latter clinical entity was only defined in 2002) meaning that the reviewed studies might have mixed both entities. In the LiS, sleep alterations are very variable and depend mainly on brainstem lesion severity. Finally, in patients recovering from coma, hypersomnia and insomnia are common phenomena. It does not appear that there are distinct defined polysomnographic patterns which clearly define each of the reviewed DOC. Additionally, there is no data indicating that sleep in DOC is still under normal circadian and homeostasic control (except for patients showing recovery of consciousness). Future studies should therefore aim to use prolonged PSG recordings together with circadian measures in well-documented patients. These tools might help to further differentiate the different DOC states, and especially the hardly distinguishable VS and MCS patients. Some studies have reported of a clear relationship between general mental ability and frontal spindle activity,69 suggesting that spindles may be different between VS and MCS. Other studies have also shown decreases in spindle density in some psychiatric conditions such as schizophrenia.70

Sleep data in DOC also appear useful for prognostic use. SC is associated with a better outcome than other coma patterns but is rarely encountered (5\% of cases27). In comatose and vegetative states, brain activity resembling standard sleep elements like spindles seem predictive of better outcome but more longitudinal sleep studies are needed in order to evaluate whether sleep (dis)organization is a reliable outcome marker in individual patients. In the LiS, no study investigated the possible prognostic value of sleep assessments.

From a methodological point of view, studying sleep in DOC is particularly challenging. Adapted and validated standardized behavioral scales (for review see ${ }^{2}$ ) are rarely used in sleep research in DOC. Prolonged PSG in clinical or rehabilitation environments is difficult because artifacts may arise from medical and nursing activities and from clinical instability of DOC patients encompassing "vegetative storms" (particularly during the acute and subacute stages of injury). All these factors make the recording of reliable EEG a real challenge in these non-collaborative severely brain damaged patients. In addition, the different studies use different PSG protocols making data comparisons difficult. Another problem is that PSG signals in DOC are often so much altered that standard sleep criteria can hardly be used for sleep stages scoring. Nevertheless, the systematic and objective evaluation of sleep might offer a promising way to better understand residual brain activity and refine diagnosis and prognosis in DOC.

\section{Practice points}

In disorders of consciousness,

1) the existence of a sleep-wake cycle as a circadian and homeostasic phenomenon has not been thoroughly investigated; 
2) the standard sleep scoring rules are of unknown applicability;

3) the available data on sleep are heterogeneous and their diagnostic and prognostic value remain unproven.

\section{Research agenda}

In the future, research in DOC should aim to:

1) use prolonged heart-rate, blood pressure, body temperature and hormonal monitoring to assess the existence of circadian control and use wake extension protocols to assess its possible homeostasic regulation;

2) define new objective markers for vigilance and sleep in prolonged polysomnographic recordings;

3) more consistently correlate sleep data with different clinical entities encountered following coma and especially their long-term prognosis.

\section{Acknowledgments}

SL and PM are respectively Senior Research Associate and Research Director at the Fonds de la Recherche Scientifique (FRS). MS was supported by the Austrian Science Fund Erwin-Schrödinger Fellowship J2470-B02. This research was funded by FRS, "Fonds pour la formation à la Recherche dans l'Industrie et dans l'Agriculture" (FRIA), European Commission (DISCOS and Mindbridge), McDonnell Foundation, Mind Science Foundation, Reine Elisabeth Medical Foundation, and University of Liège.

\section{Nomenclature}

DOC disorders of consciousness

EEG electroencephalography

fMRI functional magnetic resonance imaging

GCS Glasgow coma scale

ICU intensive care unit

LiS locked-in syndrome

MCS minimally conscious state

NREM non-rapid eye movement sleep

PLM periodic leg movement

PPT pedunculopontine tegmentum

PSG polysomnography

REM rapid eye movement sleep

SC spindle coma

SWS slow-wave sleep

VS vegetative state

\section{References}

* The Most important references are denoted by an asterisk. 
1. Laureys S, Owen AM, Schiff ND. Brain function in coma, vegetative state, and related disorders. Lancet Neurol. 2004; 3(9):537-46. [PubMed: 15324722]

2. Majerus S, Gill-Thwaites H, Andrews K, Laureys S. Behavioral evaluation of consciousness in severe brain damage. Prog Brain Res. 2005; 150:397-413. [PubMed: 16186038]

3. Brenner RP. The interpretation of the EEG in stupor and coma. Neurologist. 2005; 11(5):271-84. [PubMed: 16148734]

4. Daltrozzo J, Wioland N, Mutschler V, Kotchoubey B. Predicting coma and other low responsive patients outcome using event-related brain potentials: a meta-analysis. Clin Neurophysiol. 2007; 118(3):606-14. [PubMed: 17208048]

*5. Rechtschaffen, A.; Kales, A. A manual of standardized terminology, techniques and scoring system for sleep stages of human subjects. U.S. Dept. of Health, Education, and Welfare; Bethesda, Md.: 1968. p. 12

6. Parthasarathy S, Tobin MJ. Sleep in the intensive care unit. Intensive Care Med. 2004; 30(2):197206. [PubMed: 14564378]

7. Cabello B, Parthasarathy S, Mancebo J. Mechanical ventilation: let us minimize sleep disturbances. Curr Opin Crit Care. 2007; 13(1):20-6. [PubMed: 17198045]

8. Himanen SL, Hasan J. Limitations of Rechtschaffen and Kales. Sleep Med Rev. 2000; 4(2):149-67. [PubMed: 12531164]

9. Pattoneri P, Tirabassi G, Pela G, Astorri E, Mazzucchi A, Borghetti A. Circadian blood pressure and heart rate changes in patients in a persistent vegetative state after traumatic brain injury. $\mathrm{J}$ Clin Hypertens (Greenwich). 2005; 7(12):734-9. [PubMed: 16330896]

10. Fukudome Y, Abe I, Saku Y, Matsumura K, Sadoshima S, Utunomiya H, et al. Circadian blood pressure in patients in a persistent vegetative state. Am J Physiol. 1996; 270(5 Pt 2):R1109-14. [PubMed: 8928913]

11. Mundigler G, Delle-Karth G, Koreny M, Zehetgruber M, Steindl-Munda P, Marktl W, et al. Impaired circadian rhythm of melatonin secretion in sedated critically ill patients with severe sepsis. Crit Care Med. 2002; 30(3):536-40. [PubMed: 11990911]

12. Bergamasco B, Bergamini L, Doriguzzi T, Sacerdote I. The sleep cycle in coma: prognostic value. Electroencephalogr Clin Neurophysiol. 1968; 25(1):87. [PubMed: 4174810]

13. Chatrian GE, White LE Jr, Daly D. Electroencephalographic patterns resembling those of sleep in certain comatose states after injuries to the head. Electroencephalogr Clin Neurophysiol. 1963; 15:272-80. [PubMed: 14020346]

14. Ron S, Algom D, Hary D, Cohen M. Time-related changes in the distribution of sleep stages in brain injured patients. Electroencephalogr Clin Neurophysiol. 1980; 48(4):432-41. [PubMed: 6153604]

15. Oksenberg A, Gordon C, Arons E, Sazbon L. Phasic activities of rapid eye movement sleep in vegetative state patients. Sleep. 2001; 24(6):703-6. [PubMed: 11560184]

16. Halasz P, Terzano M, Parrino L, Bodizs R. The nature of arousal in sleep. J Sleep Res. 2004; 13(1):1-23. [PubMed: 14996030]

17. Dinges DF, Douglas SD, Hamarman S, Zaugg L, Kapoor S. Sleep deprivation and human immune function. Adv Neuroimmunol. 1995; 5(2):97-110. [PubMed: 7496616]

18. Freedman NS, Gazendam J, Levan L, Pack AI, Schwab RJ. Abnormal sleep/wake cycles and the effect of environmental noise on sleep disruption in the intensive care unit. Am J Respir Crit Care Med. 2001; 163(2):451-7. [PubMed: 11179121]

19. Evans BM. What does brain damage tell us about the mechanisms of sleep? J R Soc Med. 2002; 95(12):591-7. [PubMed: 12461144]

20. Kaplan PW. The EEG in metabolic encephalopathy and coma. J Clin Neurophysiol. 2004; 21(5): 307-18. [PubMed: 15592005]

21. Jung KY, Han SG, Lee KH, Chung CS. Repetitive leg movements mimicking periodic leg movement during sleep in a brain-dead patient. Eur J Neurol. 2006; 13(7):e3-4. [PubMed: 16834692]

22. Kim JS, Lee SB, Park SK, Han SR, Kim YI, Lee KS. Periodic limb movement during sleep developed after pontine lesion. Mov Disord. 2003; 18(11):1403-5. [PubMed: 14639696] 
23. Yokota T, Hirose K, Tanabe H, Tsukagoshi H. Sleep-related periodic leg movements (nocturnal myoclonus) due to spinal cord lesion. J Neurol Sci. 1991; 104(1):13-8. [PubMed: 1919596]

24. Nogueira de Melo A, Krauss GL, Niedermeyer E. Spindle coma: observations and thoughts. Clin Electroencephalogr. 1990; 21(3):151-61. [PubMed: 2364557]

25. Lopez MR, Freeman R, Schomer D. Spindle coma secondary to vasovagal syncope. Epilepsy Behav. 2002; 3(3):289-91. [PubMed: 12662612]

*26. Hansotia P, Gottschalk P, Green P, Zais D. Spindle coma: incidence, clinicopathologic correlates, and prognostic value. Neurology. 1981; 31(1):83-7. [PubMed: 7192831]

27. Kaplan PW, Genoud D, Ho TW, Jallon P. Clinical correlates and prognosis in early spindle coma. Clin Neurophysiol. 2000; 111(4):584-90. [PubMed: 10727908]

28. Hulihan JF Jr, Syna DR. Electroencephalographic sleep patterns in post-anoxic stupor and coma. Neurology. 1994; 44(4):758-60. [PubMed: 8164840]

*29. Cheliout-Heraut F, Rubinsztajn R, Ioos C, Estournet B. Prognostic value of evoked potentials and sleep recordings in the prolonged comatose state of children. Preliminary data. Neurophysiol Clin. 2001; 31(5):283-92. [PubMed: 11817270]

*30. Rumpl E, Prugger M, Bauer G, Gerstenbrand F, Hackl JM, Pallua A. Incidence and prognostic value of spindles in post-traumatic coma. Electroencephalogr Clin Neurophysiol. 1983; 56(5): 420-9. [PubMed: 6194958]

31. Britt CW Jr. Nontraumatic "spindle coma": clinical, EEG, and prognostic features. Neurology. 1981; 31(4):393-7. [PubMed: 7194431]

32. Britt CW Jr, Raso E, Gerson LP. Spindle coma, secondary to primary traumatic midbrain hemorrhage. Electroencephalogr Clin Neurophysiol. 1980; 49(3-4):406-8. [PubMed: 6158419]

33. Seet RC, Lim EC, Wilder-Smith EP. Spindle coma from acute midbrain infarction. Neurology. 2005; 64(12):2159-60. [PubMed: 15985602]

34. Evans BM, Bartlett JR. Prediction of outcome in severe head injury based on recognition of sleep related activity in the polygraphic electroencephalogram. J Neurol Neurosurg Psychiatry. 1995; 59(1):17-25. [PubMed: 7608703]

35. Valente M, Placidi F, Oliveira AJ, Bigagli A, Morghen I, Proietti R, et al. Sleep organization pattern as a prognostic marker at the subacute stage of post--traumatic coma. Clin Neurophysiol. 2002; 113(11):1798-805. [PubMed: 12417234]

36. Giubilei F, Formisano R, Fiorini M, Vitale A, Faroni J, Toni D, et al. Sleep abnormalities in traumatic apallic syndrome. J Neurol Neurosurg Psychiatry. 1995; 58(4):484-6. [PubMed: 7738562]

37. D'Aleo G, Saltuari L, Gerstenbrand F, Bramanti P. Sleep in the last remission stages of vegetative state of traumatic nature. Funct Neurol. 1994; 9(4):189-92. [PubMed: 7883204]

38. D'Aleo G, Bramanti P, Silvestri R, Saltuari L, Gerstenbrand F, Di Perri R. Sleep spindles in the initial stages of the vegetative state. Ital J Neurol Sci. 1994; 15(7):347-51. [PubMed: 7698892]

*39. Gordon CR, Oksenberg A. Spontaneous nystagmus across the sleep-wake cycle in vegetative state patients. Electroencephalogr Clin Neurophysiol. 1993; 86(2):132-7. [PubMed: 7681380]

40. Oksenberg A, Arons E, Sazbon L, Mizrahi A, Radwan H. Sleep-related erections in vegetative state patients. Sleep. 2000; 23(7):953-7. [PubMed: 11083604]

41. Hobson JA. How the brain goes out of its mind. Endeavour. 1996; 20(2):86-9. [PubMed: 8690003]

42. Shouse MN, Siegel JM. Pontine regulation of REM sleep components in cats: integrity of the pedunculopontine tegmentum (PPT) is important for phasic events but unnecessary for atonia during REM sleep. Brain Res. 1992; 571(1):50-63. [PubMed: 1611494]

43. Kampfl A, Schmutzhard E, Franz G, Pfausler B, Haring HP, Ulmer H, et al. Prediction of recovery from post-traumatic vegetative state with cerebral magnetic-resonance imaging. Lancet. 1998; 351(9118):1763-7. [PubMed: 9635948]

*44. Isono M, Wakabayashi Y, Fujiki MM, Kamida T, Kobayashi H. Sleep cycle in patients in a state of permanent unconsciousness. Brain Inj. 2002; 16(8):705-12. [PubMed: 12167195]

45. Giacino JT, Ashwal S, Childs N, Cranford R, Jennett B, Katz DI, et al. The minimally conscious state: definition and diagnostic criteria. Neurology. 2002; 58(3):349-53. [PubMed: 11839831] 
46. George B, Landau-Ferey J, Benoit O, Dondey M, Cophignon J, Cohen M, Oksenberg A, Snir D, Stern MJ, Groswasser Z. Night sleep disorders during recovery of severe head injuries [author's transl]. Neurochirurgie. 1981; 27(1):35-8. [PubMed: 7254451]

47. George B, Landau-Ferey J. Twelve months' follow-up by night sleep EEG after recovery from severe head trauma. Neurochirurgia (Stuttg). 1986; 29(2):45-7. [PubMed: 3713952]

48. Keshavan MS, Channabasavanna SM, Reddy GN. Post-traumatic psychiatric disturbances: patterns and predictors of outcome. Br J Psychiatry. 1981; 138:157-60. [PubMed: 7260498]

49. Cohen M, Oksenberg A, Snir D, Stern MJ, Groswasser Z. Temporally related changes of sleep complaints in traumatic brain injured patients. J Neurol Neurosurg Psychiatry. 1992; 55(4):313-5. [PubMed: 1583518]

50. Clinchot DM, Bogner J, Mysiw WJ, Fugate L, Corrigan J. Defining sleep disturbance after brain injury. Am J Phys Med Rehabil. 1998; 77(4):291-5. [PubMed: 9715917]

*51. Guilleminault C, Yuen KM, Gulevich MG, Karadeniz D, Leger D, Philip P. Hypersomnia after head-neck trauma: a medicolegal dilemma. Neurology. 2000; 54(3):653-9. [PubMed: 10680799]

*52. Masel BE, Scheibel RS, Kimbark T, Kuna ST. Excessive daytime sleepiness in adults with brain injuries. Arch Phys Med Rehabil. 2001; 82(11):1526-32. [PubMed: 11689971]

53. Fichtenberg NL, Millis SR, Mann NR, Zafonte RD, Millard AE. Factors associated with insomnia among post-acute traumatic brain injury survivors. Brain Inj. 2000; 14(7):659-67. [PubMed: 10914647]

54. Fichtenberg NL, Zafonte RD, Putnam S, Mann NR, Millard AE. Insomnia in a post-acute brain injury sample. Brain Inj. 2002; 16(3):197-206. [PubMed: 11874613]

55. Ouellet MC, Savard J, Morin CM. Insomnia following traumatic brain injury: a review. Neurorehabil Neural Repair. 2004; 18(4):187-98. [PubMed: 15669131]

56. Ouellet MC, Morin CM. Subjective and objective measures of insomnia in the context of traumatic brain injury: a preliminary study. Sleep Med. 2006; 7(6):486-97. [PubMed: 16934524]

57. Ouellet MC, Beaulieu-Bonneau S, Morin CM. Insomnia in patients with traumatic brain injury: frequency, characteristics, and risk factors. J Head Trauma Rehabil. 2006; 21(3):199-212. [PubMed: 16717498]

58. Ouellet MC, Morin CM. Cognitive behavioral therapy for insomnia associated with traumatic brain injury: a single-case study. Arch Phys Med Rehabil. 2004; 85(8):1298-302. [PubMed: 15295756]

59. Hoedlmoser K, Pecherstorfer T, Gruber G, Anderer P, Doppelmayr M, Klimesch W, et al. Instrumental conditioning of human sensorimotor rhythm $(12-15 \mathrm{~Hz})$ and its impact on sleep as well as declarative learning. Sleep. 2008; 31(10):1401-8. [PubMed: 18853937]

*60. Guilleminault C, Cathala JP, Castaigne P. Effects of 5-hydroxytryptophan on sleep of a patient with a brain-stem lesion. Electroencephalogr Clin Neurophysiol. 1973; 34(2):177-84. [PubMed: 4119531]

61. Markand ON, Dyken ML. Sleep abnormalities in patients with brain stem lesions. Neurology. 1976; 26(8):769-76. [PubMed: 945872]

62. Cummings JL, Greenberg R. Sleep patterns in the "locked-in" syndrome. Electroencephalogr Clin Neurophysiol. 1977; 43(2):270-1. [PubMed: 69537]

63. Freemon FR, Salinas-Garcia RF, Ward JW. Sleep patterns in a patient with a brain stem infarction involving the raphe nucleus. Electroencephalogr Clin Neurophysiol. 1974; 36(6):657-60. [PubMed: 4135364]

64. Baldy-Moulinier M, Billiard M, Escuret E, Roquefeuil B, Fuentes JM, Passouant P. A polygraphic, haemodynamic and metabolic study of 2 cases of the "locked-in" syndrome. Rev Electroencephalogr Neurophysiol Clin. 1977; 7(4):473-8. [author's trans1]. [PubMed: 611595]

*65. Autret A, Laffont F, de Toffol B, Cathala HP. A syndrome of REM and non-REM sleep reduction and lateral gaze paresis after medial tegmental pontine stroke. Computed tomographic scans and anatomical correlations in four patients. Arch Neurol. 1988; 45(11):1236-42. [PubMed: 3190504]

66. Tamura K, Karacan I, Williams RL, Meyer JS. Disturbances of the sleep-waking cycle in patients with vascular brain stem lesions. Clin Electroencephalogr. 1983; 14(1):35-46. [PubMed: 6831733]

67. Lavie P, Pratt H, Scharf B, Peled R, Brown J. Localized pontine lesion: nearly total absence of REM sleep. Neurology. 1984; 34(1):118-20. [PubMed: 6537835] 
68. Oksenberg A, Soroker N, Solzi P, Reider-Groswasser I. Polysomnography in locked-in syndrome. Electroencephalogr Clin Neurophysiol. 1991; 78(4):314-7. [PubMed: 1706254]

69. Bodizs R, Kis T, Lazar AS, Havran L, Rigo P, Clemens Z, et al. Prediction of general mental ability based on neural oscillation measures of sleep. J Sleep Res. 2005; 14(3):285-92. [PubMed: 16120103]

70. Ferrarelli F, Massimini M, Peterson MJ, Riedner BA, Lazar M, Murphy MJ, et al. Reduced evoked gamma oscillations in the frontal cortex in schizophrenia patients: a TMS/EEG study. Am J Psychiatry. 2008; 165(8):996-1005. [PubMed: 18483133] 


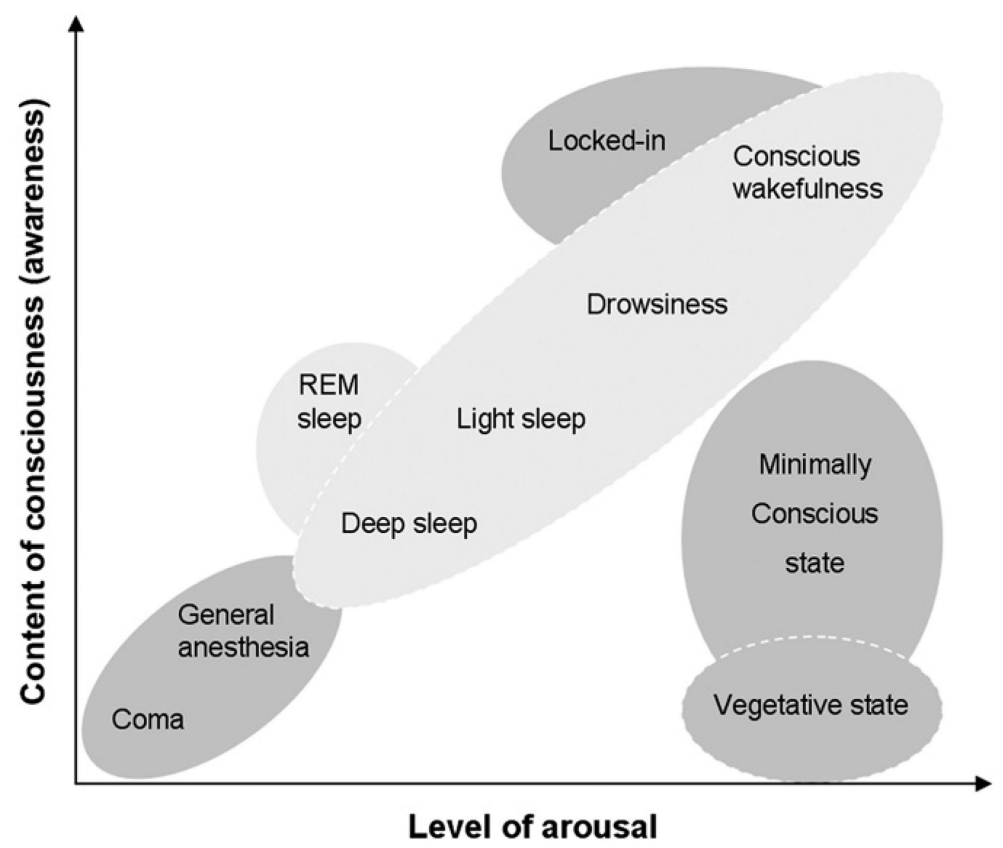

Fig. 1.

Simplified illustration of the two main components of consciousness: arousal (or wakefulness) and the content of consciousness (i.e. awareness). In normal physiological states, arousal and awareness are positively correlated (with the exception of dream activity during REM-sleep). Patients in coma or under general anesthesia are unconscious because they cannot be awakened. Patients in the vegetative or the minimally conscious state are awake but have no or low awareness. Locked-in patients are awake and aware but are nearly completely paralyzed and may be mistaken for coma or vegetative state. Adapted from Laureys et al., TICS 2005. 


\section{Table 1}

The arousal (Evans et al., 1995, left column) and sleep (Valente et al., 2002, right column) scales in coma.

\begin{tabular}{ll}
\hline Arousal scale & Sleep scale \\
\hline $\begin{array}{l}\text { 1) No spontaneous arousal activity } \\
\text { 2) Abnormal spontaneous arousal activity without EEG } \\
\text { changes }\end{array}$ & $\begin{array}{l}\text { 1) Monophasic: continuous low voltage theta-delta activity } \\
\text { 2) Cyclic alternating pattern: absence of sleep elements in the presence } \\
\text { of recurrent abnormal activity }\end{array}$ \\
$\begin{array}{ll}\text { 3) Phasic activity related to abnormal spontaneous } \\
\text { arousal including EEG changes }\end{array}$ & $\begin{array}{l}\text { 3) Rudimentary sleep: presence of rudimentary NREM sleep elements } \\
\text { (spindles, K-complexes) }\end{array}$ \\
$\begin{array}{ll}\text { 4) Sleep-like traces with K-complexes responsive to } \\
\text { stimulation }\end{array}$ & $\begin{array}{l}\text { 4) NREM sleep: presence of well-structured elements of NREM sleep } \\
\text { 5) Wakeful traces with normal alpha in at least one } \\
\text { hemisphere }\end{array}$ \\
\end{tabular}

The arousal patterns 3-4 indicate good outcome whereas pattern 1 indicates bad outcome.

The sleep patterns 3-5 indicate good outcome whereas patterns 1-2 indicates bad outcome. 


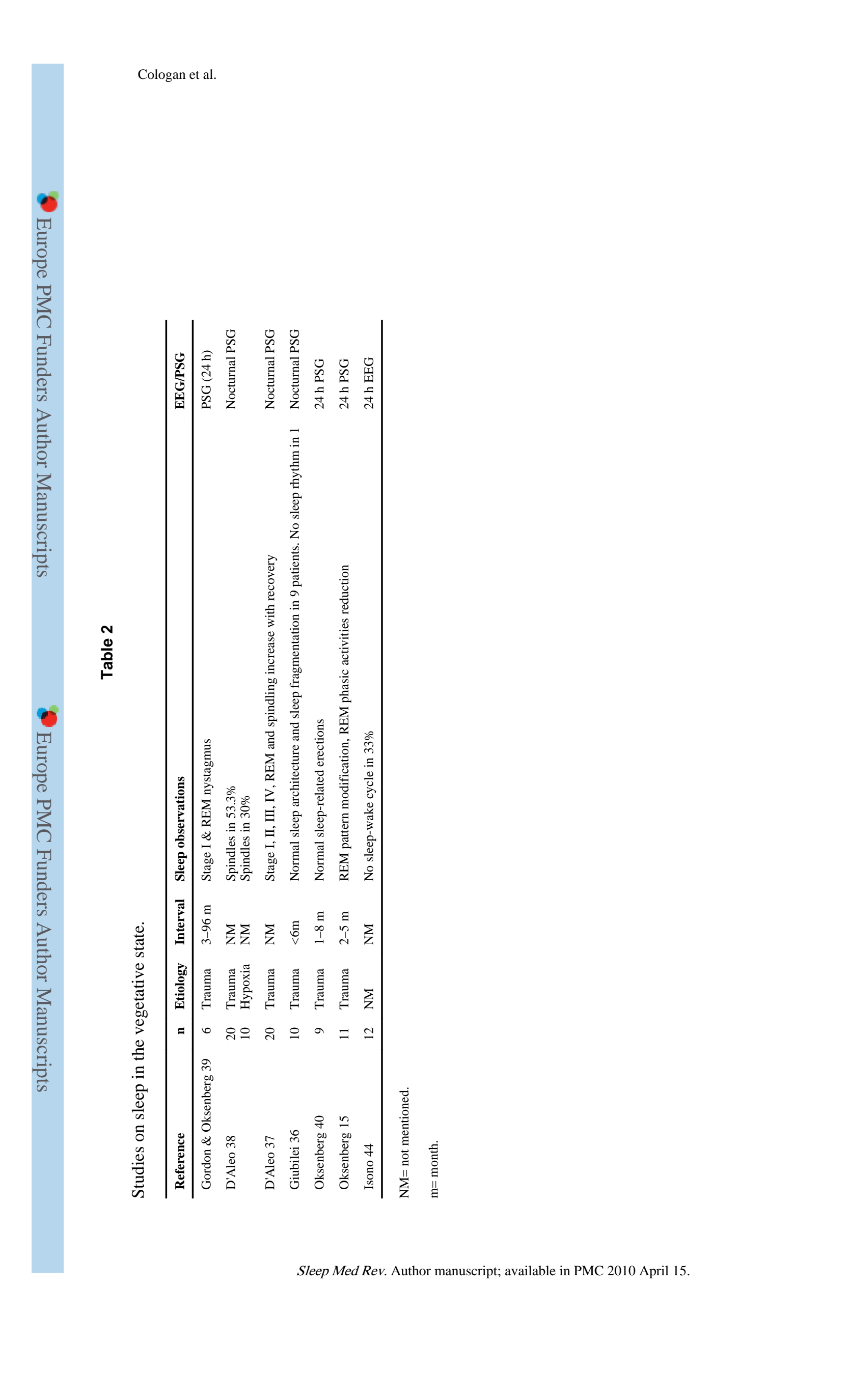




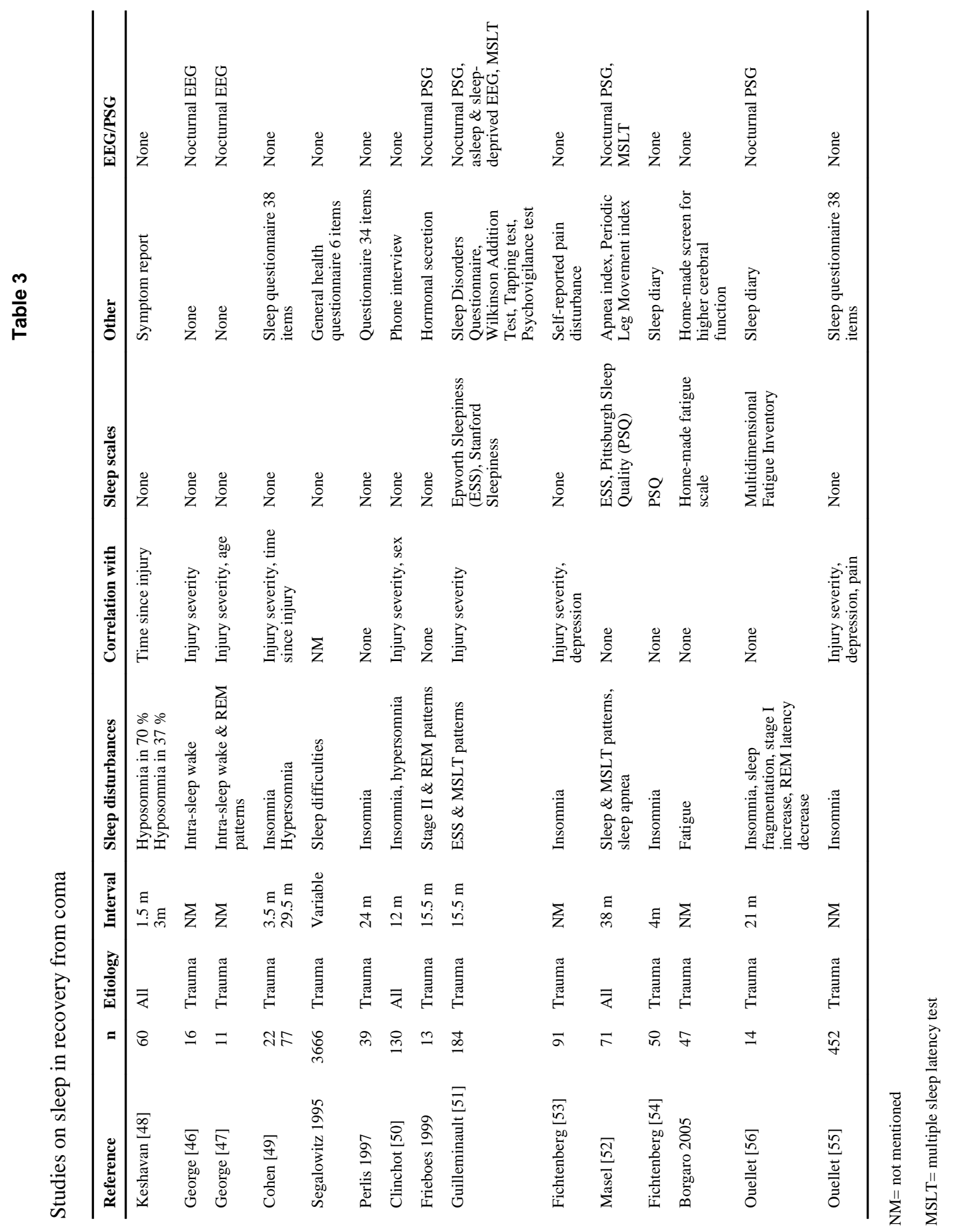




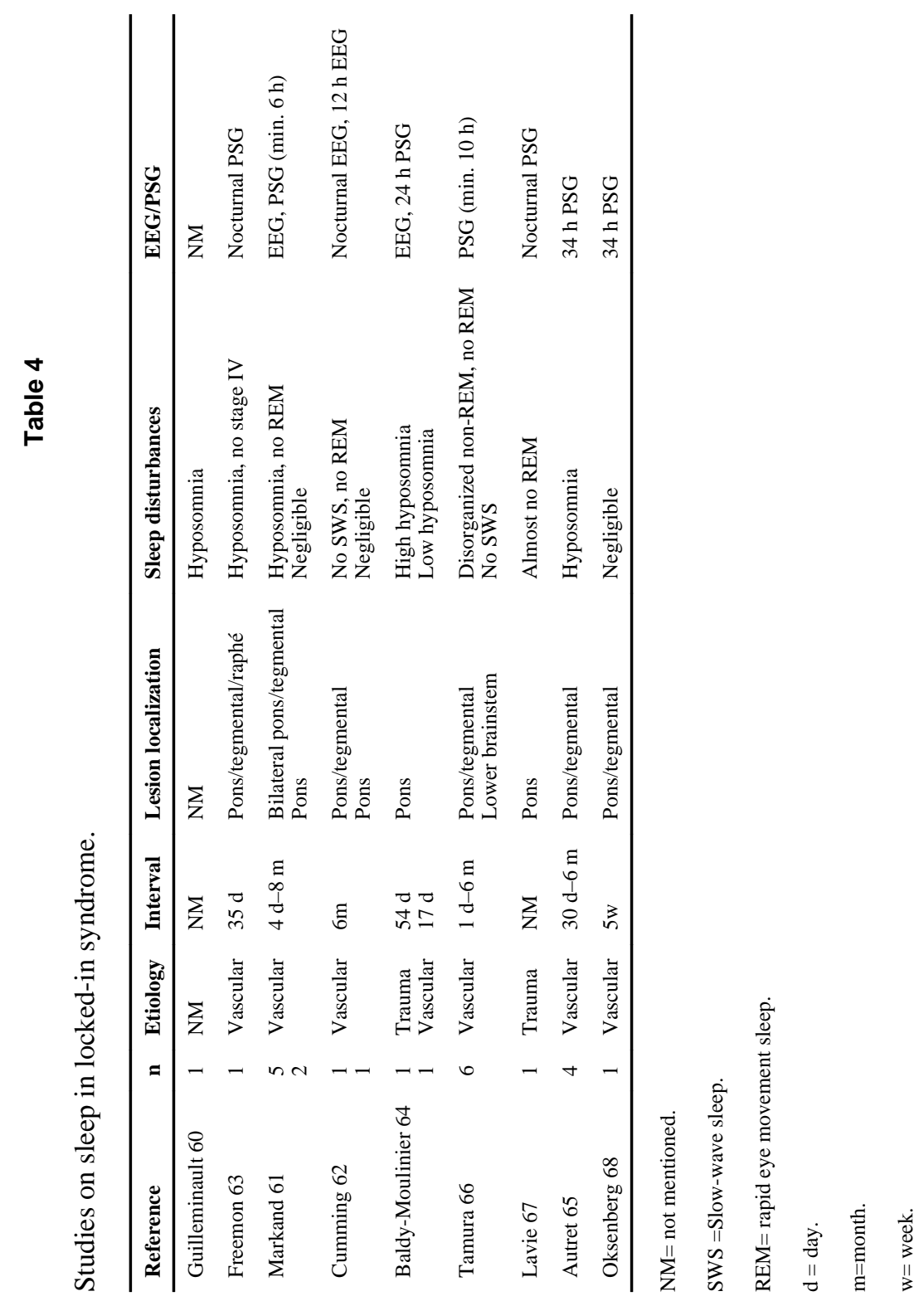

Sleep Med Rev. Author manuscript; available in PMC 2010 April 15. 